\title{
The use of antibiotics in the intensive care unit of a tertiary hospital in Malawi
}

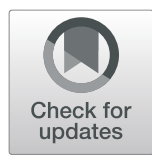

Raphael Kazidule Kayambankadzanja ${ }^{1,2^{*}+}$ D , Moses Lihaka ${ }^{2 \dagger}$, Andreas Barratt-Due ${ }^{3}$, Mtisunge Kachingwe ${ }^{1,2}$, Wezzie Kumwenda', Rebecca Lester ${ }^{4,5}$, Sithembile Bilima ${ }^{5}$, Jaran Eriksen ${ }^{6,7}$ and Tim Baker ${ }^{1,2,6,8}$

\begin{abstract}
Background: Antibiotic resistance is on the rise. A contributing factor to antibiotic resistance is the misuse of antibiotics in hospitals. The current use of antibiotics in ICUs in Malawi is not well documented and there are no national guidelines for the use of antibiotics in ICUs. The aim of the study was to describe the use of antibiotics in a Malawian ICU.

Methods: A retrospective review of medical records of all admissions to the main ICU in Queen Elizabeth Central Hospital in Blantyre, Malawi, between January 2017 and April 2019. Data were extracted from the ICU patient register on clinical parameters on admission, diagnoses, demographics and antibiotics both prescribed and given for all patients admitted to the ICU. Usage of antibiotics in the ICU and bacterial culture results from samples taken in the ICU and in the peri-ICU period, (from 5 days before ICU admission to 5 days after ICU discharge), were described.
\end{abstract}

Results: Six hundred-and-forty patients had data available on prescribed and received medications and were included in the analyses. Of these, 577 (90.2\%) were prescribed, and 522 (81.6\%) received an antibiotic in ICU. The most commonly used antibiotics were ceftriaxone, given to $470(73.4 \%)$ of the patients and metronidazole to 354 (55.3\%). Three-hundred-and-thirty-three (52.0\%) of the patients received more than one type of antibiotic concurrently - ceftriaxone and metronidazole was the most common combination, given to 317 patients. Forty five patients (7.0\%) were given different antibiotics sequentially. One-hundred-and-thirty-seven patients (21.4\%) had a blood culture done in the peri-ICU period, of which 70 (11.0\% of the patients) were done in the ICU. Twenty-five (18.3\%) of the peri-ICU cultures were positive and eleven different types of bacteria were grown in the cultures, of which $17.2 \%$ were sensitive to ceftriaxone.

Conclusion: We have found a substantial usage of antibiotics in an ICU in Malawi. Ceftriaxone, the last-line antibiotic in the national treatment guidelines, is commonly used, and bacteria appear to show high levels of resistance to it, although blood culture testing is infrequently used. Structured antibiotic stewardship programs may be useful in all ICUs.

Keywords: Antibiotics, Antibiotic resistance, ICU, Malawi, Africa

\footnotetext{
* Correspondence: raphkazidule@gmail.com

Raphael Kazidule Kayambankadzanja and Moses Lihaka joint First Authors

'Department of Anaesthesia and Intensive Care, Queen Elizabeth Central

Hospital, Blantyre, Malawi

${ }^{2}$ College of Medicine, University of Malawi, Blantyre, Malawi

Full list of author information is available at the end of the article
}

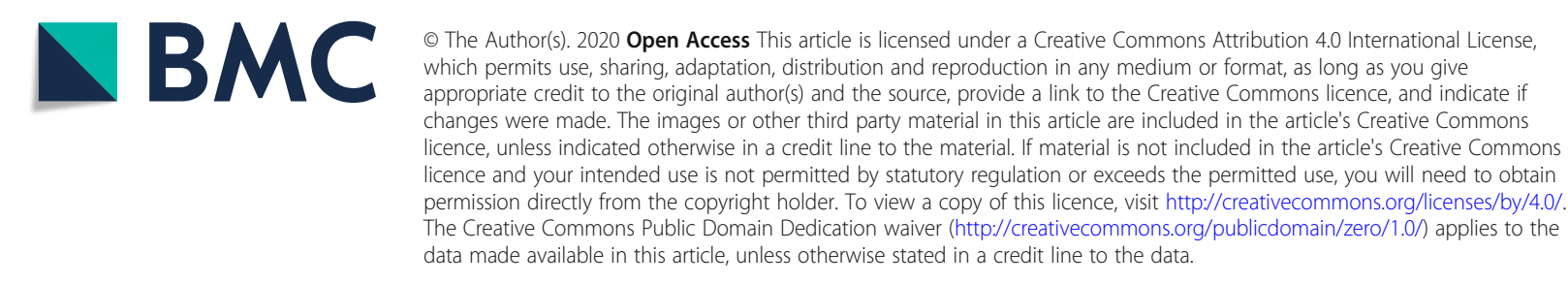




\section{Introduction}

Antibiotic resistance is on the rise. Globally, the estimated 700,000 people who die each year due to drug resistant infections is projected to increase to 10 million a year by 2050 [1]. The rate of production of new antibiotics is declining [2] and the UN states that resistance to antibiotics is a global health threat requiring urgent attention $[3,4]$.

A contributing factor to antibiotic resistance is the misuse of antibiotics in hospitals [5-7]. A recent study in Malawi found that one-third of bacteria grown in blood cultures from hospitalized children were resistant to Ceftriaxone, the most commonly used parenteral antibiotic in the hospital [8]. Resistance additionally results in longer hospital stays for patients [9], increased costs [10] and death [11].

A rational use of antibiotics is necessary for optimizing patient outcomes [11]. Critically ill patients admitted to Intensive Care Units (ICUs) are at greater risk of serious morbidity and death if antibiotic therapy fails [11-13]. Malawi, a low-income country in Africa, has 25 ICU beds for a population of 17 million people [14, 15]. The current use of antibiotics in ICUs in Malawi is not well documented and there are no national guidelines for the use of antibiotics in ICUs. The aim of the study was to describe the use of antibiotics in a Malawian ICU.

\section{Methods}

A retrospective review of medical records for all admissions to the main ICU in Queen Elizabeth Central Hospital in Blantyre, Malawi between January 2017 and April 2019.

\section{Study setting}

Queen Elizabeth Central Hospital (QECH) is a large, state-run hospital in Blantyre, Malawi with 1000 beds. The hospital serves an immediate catchment population of 1 million people and is a referral center for the southern region of Malawi and the whole country. The main ICU has four beds and admits patients from all wards and all specialties in the hospital. The closed ICU is run by the department of anesthesia and intensive care and has continuous observation with a 1:1 nurse: patient ratio, vital signs monitoring, oxygen supplied by cylinders, electric suction, mechanical ventilation for the four beds, and can deliver vasoactive infusions with syringe drivers.

\section{Study population}

All patients admitted to the ICU between January 2017 and April 2019. Patients who lacked data on prescribed and received medications were excluded.

\section{Data collection}

Data for the study were extracted from the ICU patient register. The register contains information on clinical parameters at admission, diagnoses, demographics and treatments both prescribed and given, (some patients were prescribed a treatment by clinicians, but were not given the treatment due to stock-outs or other reasons), for all patients admitted to the ICU. Medication prescriptions in the ICU are primarily done by the admitting anesthetic clinical officer or anesthesiologist often in consultation with other specialty physicians. The specialty team the patients belongs to at times reviews prescriptions and can modify in agreement with the anaesthetist in the ICU. Admission data were extracted to a paper-based data collection tool from the patient observation charts by the research team from January 2017 to November 2017, and by the nurses and clinicians in the ICU from December 2017 onwards. Data on antibiotic treatments during the ICU period were added to the register from patient treatment charts and blood culture results were added to the register from the hospital's laboratory database, which is run by the neighboring research affiliate, the Malawi-Liverpool-Wellcome Trust. Other types of cultures are not done in the hospital. The laboratory uses bioMérieux BACT/ALERT 3D for detecting microorganisms in blood. Routine standard laboratory procedures were followed by qualified laboratory personnel and antibiotic sensitivity profiles interpreted according to British Society for antimicrobial chemotherapy guidelines. Quality controls are performed using known Public Health of England National Collection of Type Cultures bacterial reference strains and the laboratory participates in external UK and USA based quality assurance. The data were entered into the electronic register database using double-data-entering and quality-checking by two staff members, and follow-up of missing data by the ICU clerk. An anonymous extract of the data was used for the study.

\section{Data analysis}

Descriptive data were summarized using proportions, means, ranges, medians and interquartile ranges where appropriate. The many diverse diagnoses were grouped into eight categories, (serious infection, noncommunicable disease [e.g. cancer, anaemia, unspecified tumours], trauma, bowel perforation or obstruction, post-delivery or abortion care, acute respiratory disease, pre-eclampsia/eclampsia, other/unknown), by the researchers. The number of antibiotics given to each patient was recorded, and the usage of more than one antibiotic was classed as concurrent or sequential. Concurrent use was a patient receiving two or more antibiotics at the same time. Sequential use was when the first antibiotic was discontinued and subsequently a second 
antibiotic was commenced. Blood cultures were classified as being taken on the first day of admission to ICU, taken during the ICU stay, taken during the peri-ICU period - from 5 days before ICU admission to 5 days after ICU discharge - or taken at any point during the hospital stay. Blood culture results were classified as positive or negative, and laboratory results stating, "no significant growth (contaminant)", were classified as negative. Sensitivities to ceftriaxone of the cultured bacteria were noted. Data analysis was done with STATA (Release 15, StataCorp, College Station, TX). Ethical clearance was granted by the College of Medicine Research and Ethics Committee (COMREC P.07/18/2433).

\section{Results}

There was a total of 740 admissions to the ICU during the study period. Six hundred and forty patients had information on prescribed and received medications and were included in the analysis. Their median age was 32 (IQR 22-45) and 326 (51.0\%) were male. The surgical and medical departments contributed more than half the total number of participants with 213 (33.3\%) and 131 (20.5\%) respectively. Post-operative admissions accounted for $352(55.0 \%)$ of the participants. The participants' median length of stay in ICU was 2 days (IQR $1-4), 34.8 \%$ died in-ICU and a total of $45.5 \%$ died inhospital. (Table 1). The characteristics of the one hundred patients who were excluded due to missing information about medications did not differ substantially from the included participants (Supplementary Table 1).

\section{Antibiotic usage in ICU}

Of the 640 patients, $522(81.6 \%)$ were given an antibiotic. The modal number of antibiotics received was two and $18(2.8 \%)$ received four or five different antibiotics during their stay in ICU (Fig. 1). In total, 577 patients (90.2\%) were prescribed an antibiotic and fifty-five (9.5\%) of the patients were prescribed but did not receive the antibiotic.

The most commonly used antibiotic was ceftriaxone, given to $470(73.4 \%)$ of the patients. The standard dose of ceftriaxone to patients with normal renal function was $2 \mathrm{~g}$, given once a day - the frequency increased to twice a day in patients with meningitis. Metronidazole was given to 354 (55.3\%) and meropenem to 30 (4.7\%) patients (Table 2). Three-hundred-and-thirty-three (52.0\%) of the participants received more than one type of antibiotic concurrently - ceftriaxone and metronidazole was the most common combination, given to 317 patients (49.5\%), 58 patients $(9.1 \%)$ received a different combination. Forty five patients $(7.0 \%)$ were given different antibiotics sequentially.
Table 1 Patient and Infection characteristics

\begin{tabular}{|c|c|}
\hline Variable & $\begin{array}{l}\mathrm{n}(\%) \\
N=640\end{array}$ \\
\hline Male sex & $326(51.0 \%)$ \\
\hline Age in yrs. Median (IQR) & $32(22-45)$ \\
\hline \multicolumn{2}{|l|}{ Specialty } \\
\hline Surgery & $213(33.3 \%)$ \\
\hline Medicine & $131(20.5 \%)$ \\
\hline Obstetrics and Gynaecology & $108(16.9 \%)$ \\
\hline Neurosurgery & $105(16.4 \%)$ \\
\hline Peadiatrics & $39(6.1 \%)$ \\
\hline Ear, Nose and Throat & $19(3.0 \%)$ \\
\hline Other $^{a}$ & $25(4.0 \%)$ \\
\hline \multicolumn{2}{|l|}{ Admitted from } \\
\hline Theatre & $352(55.0 \%)$ \\
\hline Emergency department & $137(21.4 \%)$ \\
\hline Ward & $128(20.0 \%)$ \\
\hline Recovery room & $3(0.5 \%)$ \\
\hline Other & $20(3.1 \%)$ \\
\hline \multicolumn{2}{|l|}{ Diagnosis } \\
\hline Serious Infection ${ }^{b}$ & $141(22.0 \%)$ \\
\hline Non-communicable disease ${ }^{c}$ & $133(20.8 \%)$ \\
\hline Trauma $^{d}$ & $103(16.1 \%)$ \\
\hline Bowel perforation or obstruction ${ }^{e}$ & $98(15.3 \%)$ \\
\hline Post-delivery or abortion care & $43(6.7 \%)$ \\
\hline Acute respiratory disease $^{f}$ & $35(5.5 \%)$ \\
\hline Pre-eclampsia/eclampsia & $16(2.5 \%)$ \\
\hline Other/ Unknown & $71(11.1 \%)$ \\
\hline Length of stay in days Median (IQR) & $2(1-4)$ \\
\hline Duration of care with antibiotics in the ICU Median (IQR) & $2(1-4)$ \\
\hline ICU mortality & $223(34.8 \%)$ \\
\hline Length of stay for those who died Median (IQR) & $2(1-5)$ \\
\hline Hospital Mortality & $291(45.5 \%)$ \\
\hline
\end{tabular}

a Orthopedics, Burns and Oncology

bIncluding Meningitis, Malaria, Endometritis, Pneumonia, sepsis/septic shock and Tuberculosis

' Cancer, Anaemia, unspecified tumours

${ }^{\mathrm{I}}$ Including Head injury and Burns

e including Typhoid perforation

${ }^{f}$ includes Asthma and Pulmonary Oedema

\section{Blood cultures}

In total, 192 (30.0\% of the patients) had a blood culture done during their stay in-hospital. One-hundred-andthirty seven- (21.4\% of the patients) had a blood culture done in the peri-ICU period, of these 70 were done in the ICU (11.0\% of the patients) and 9 (12.9\% of the ICU cultures) were done in ICU on the day of admission. Of the eleven patients with positive ICU cultures, nine were treated with ceftriaxone. In five of these, bacteria were grown that were resistant to ceftriaxone. All the five 
60.0

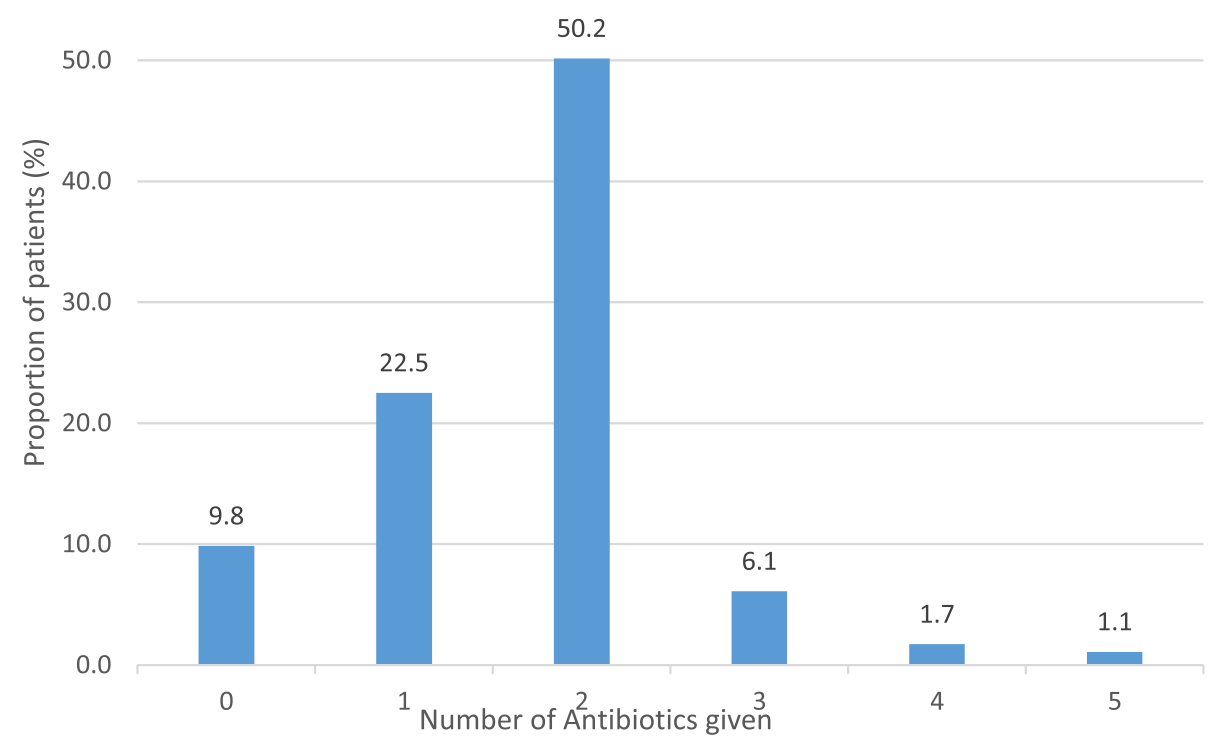

Fig. 1 Number of antibiotics given to the patients in ICU

Table 2 Antibiotics prescribed and given in the ICU

\begin{tabular}{|c|c|c|c|c|}
\hline Antibiotic & $\begin{array}{l}\text { Number of courses } \\
\text { prescribed } N=1024\end{array}$ & $\begin{array}{l}\text { Number of courses } \\
\text { given } N=933\end{array}$ & $\begin{array}{l}\text { Proportion of all } \\
\text { antibiotic courses } \\
\text { given } N=933\end{array}$ & $\begin{array}{l}\text { Proportion of patients } \\
\text { who were given the } \\
\text { antibiotic } N=640\end{array}$ \\
\hline Ceftriaxone & 520 & 470 & $50.4 \%$ & $73.4 \%$ \\
\hline Metronidazole & 378 & 354 & $38.0 \%$ & $55.3 \%$ \\
\hline Meropenem & 43 & 30 & $3.2 \%$ & $4.7 \%$ \\
\hline Ciprofloxacin & 17 & 17 & $1.8 \%$ & $2.6 \%$ \\
\hline Cotrimoxazole & 15 & 12 & $1.3 \%$ & $1.9 \%$ \\
\hline Piperacillin Tazobactam & 13 & 13 & $1.4 \%$ & $2.0 \%$ \\
\hline Gentamycin & 13 & 13 & $1.4 \%$ & $2.0 \%$ \\
\hline Doxycycline & 4 & 4 & $0.4 \%$ & $0.6 \%$ \\
\hline Amoxicillin Clavulanate & 3 & 3 & $0.3 \%$ & $0.5 \%$ \\
\hline Benzyl penicillin & 3 & 3 & $0.3 \%$ & $0.5 \%$ \\
\hline Azithromycin & 3 & 3 & $0.3 \%$ & $0.5 \%$ \\
\hline Flucoxacillin & 2 & 2 & $0.2 \%$ & $0.3 \%$ \\
\hline Amikacin & 2 & 2 & $0.2 \%$ & $0.3 \%$ \\
\hline Erythromycin & 2 & 2 & $0.2 \%$ & $0.3 \%$ \\
\hline Amoxicillin & 1 & 0 & $0.0 \%$ & $0.0 \%$ \\
\hline Ampicillin & 1 & 1 & $0.1 \%$ & $0.2 \%$ \\
\hline Ceftazidime & 1 & 1 & $0.1 \%$ & $0.2 \%$ \\
\hline Chloramphenicol & 1 & 1 & $0.1 \%$ & $0.2 \%$ \\
\hline Clindamycin & 1 & 1 & $0.1 \%$ & $0.2 \%$ \\
\hline Cloxacillin & 1 & 1 & $0.1 \%$ & $0.2 \%$ \\
\hline
\end{tabular}


patients received ceftriaxone before the blood culture was taken and no patient received ceftriaxone after resistance was found.

Of the 137 peri-ICU cultures done, 25 (18.3\%) were positive, twenty three $(16.8 \%)$ had a missing result, and $89(65.0 \%)$ were negative. Of the 70 blood cultures taken in ICU, $11(15.7 \%)$ tested positive. Among the patients who had a blood culture done in the peri-ICU period, $43.8 \%$ died while among those who did not have a blood culture done, $32.4 \%$ died.

Twenty-nine bacteria were grown in the 25 positive peri-ICU blood cultures and can be seen in Table 3. Five bacteria (17.2\%) were sensitive to ceftriaxone, (three of the Staphylococcus aureus, one of the Escherichia coli and the Salmonella typhi). Eight were not tested, and the remaining 16 were resistant to ceftriaxone, (five of the Klebsiella pnemoniae, five of the Acinetobacter baumanii, three of the Escherichia coli, two of Alpha-haemolytic streptococcus, and one of the Proteus mirabilis).

\section{Discussion}

Among a population of patients in an ICU in Malawi, 81.6\% received antibiotics during their ICU stay and more than half received two or more antibiotics. An additional $9.5 \%$ of patients were prescribed but did not receive an antibiotic - possibly due to medication stockouts.

A frequent use of antibiotics in ICUs has been reported elsewhere $[11,16,17]$. In South Africa and Ghana, 75 and $71 \%$ of ICU patients were given antibiotics respectively $[17,18]$. In our study we found ceftriaxone was the most commonly used antibiotic. This use of ceftriaxone is likely to be guideline triggered as the Malawi standard treatment guidelines (supplementary file 1) recommends that ceftriaxone be given as the initial course before a blood culture is done [19]. Cephalosporins and other broad spectrum antibiotics are also commonly used in ICUs in other countries [16, 20].
We have found a substantial use of more than one antibiotic concurrently. Some of this is likely to be rational for example the most common combination was ceftriaxone and metronidazole which, with their differing spectrum of action are a rational choice for diseases such as abdominal sepsis. Irrational concurrent use may also have been prevalent but is difficult to judge due to the lack of available detailed clinical information. Irrational use of antibiotics has been reported to be a contributing factor to poor outcomes such as prolonged length of stay and death in ICU [21] and increases costs [22]. Indeed, efforts through antibiotic stewardship programs are being put in place to help combat the problem of irrational antibiotic use in healthcare both in Malawi and other countries [13, 23-26]. During this study period, there was no evidence of such programs in the ICU and we are not aware of programs tackling antibiotic use specifically in ICUs in Malawi.

While antibiotics in ICU are used for many types and sources of infections, blood cultures may be used to direct the appropriate choice of antibiotic [11, 27, 28]. Our findings of infrequent testing of blood cultures, the low yield of positive results and the predominant use of broadspectrum antibiotics suggest that most antibiotics were being used empirically for patients with suspected infection, without the guidance of blood cultures. Despite the sparse positive blood culture results, we have found antibiotic resistance in the ICU. These findings are similar to other studies done in other ICUs in Africa [13, 29]. Resistance to ceftriaxone poses a major threat to the critically ill patients as other potent antibiotics for critically ill patients can be scarce in Malawi. The sparse use of blood cultures in the ICU, could be attributed to cost concerns, a lack of specimen collection bottles in the unit, a lack of awareness among clinicians, the fact that antibiotics are often initiated before admission to ICU so cultures may be seen as less clinically relevant, or poor communication with referring units about previous cultures and the need for repeat tests in the ICU.

Table 3 Bacteria grown in the cultures

\begin{tabular}{lll}
\hline Bacteria & Bacteria grown in the Peri-ICU positive cultures $\mathbf{N = 2 9}$ & ICU cultures with positive growth of bacteria $\mathbf{N = 1 3}$ \\
\hline Klebsiella pnemoniae & 5 & 2 \\
Acinetobacter baumanii & 5 & 4 \\
Escherichia coli & 4 & 0 \\
Alpha-haemolytic streptococcus & 4 & 2 \\
Enterococcus faecalis & 3 & 1 \\
Group D streptococcus & 2 & 2 \\
Staphylococcus aureus & 2 & 0 \\
Proteus mirabilis & 1 & 0 \\
Salmonella typhi & 1 & 0 \\
Streptococcus pyogenes & 1 & 0 \\
Pseudomonas aeruginosa & 1 & 1 \\
\hline
\end{tabular}


The higher mortality seen in patients who had blood cultures taken may seem surprising. However, it is likely explained by local practices of taking blood cultures in more severely unwell patients, or in those who are not responding to treatment.

Our study is the first of its kind to look at the antibiotic usage in an ICU in Malawi, and links over 2 years of data from the ICU register to the laboratory database. Limitations of our single-center study include the lack of available medication data for some ICU patients and the limited available clinical information to understand the rationality of the antibiotic usage.

A structured antibiotic stewardship program focusing on what is feasible may be useful in ICUs in Malawi and other low-resource settings. Patterns of infectious diseases in the unit, current treatments and outcomes for the patients should be mapped out. Local susceptibility patterns should be studied and used to develop guidelines for initial antibiotic therapy for patients with suspected bacterial infections depending on diagnosis and clinical condition. Patients' previous investigations, blood culture results and antibiotic treatments should be communicated between hospital departments. Where possible, blood cultures should be done on arrival to ICU, when a bacterial infection is suspected, and when a patient is not responding to treatment and should include sensitivity testing. Antibiotic therapy should be reviewed daily, and clinical and laboratory information used for decisions about whether to continue, modify, or discontinue therapy. Multidisciplinary teams should be involved in decisions, including ICU clinicians, nurses, specialty doctors and microbiologists [26].

\section{Conclusion}

We have found a substantial usage of antibiotics in an Intensive Care Unit in Malawi. Ceftriaxone, the last-line antibiotic in the national treatment guidelines, is commonly used, and bacteria appear to show high levels of resistance to it, although blood culture testing is infrequently used. Structured antibiotic stewardship program may be useful in all ICUs.

\section{Supplementary information}

The online version contains supplementary material available at https://doi. org/10.1186/s12879-020-05505-6.

Additional file 1: Supplementary Table 1. Characteristics of patients who were excluded due to missing information about medications in ICU. (DOCX $12 \mathrm{~kb}$ )

Additional file 2: Supplementary Table 2. Diagnoses and outcomes of the 25 patients with positive blood cultures in ICU. (DOCX $17 \mathrm{~kb}$ )

Additional file 3. Summary of Malawi Standard Treatment Guidelines. (DOCX $13 \mathrm{~kb}$ )

\section{Abbreviations}

COMREC: College of Medicine Research and Ethics Committee; ICU: Intensive Care Unit; IQR: Interquartile Range; QECH: Queen Elizabeth Central Hospital; WHO: World Health Organisation

\section{Acknowledgements}

Special thanks to Mr. Peace Morrison Kamanga for his assistance with guidelines used at Malawi Liverpool Wellcome Trust laboratory.

\section{Authors' contributions}

RKK, ML and TB conceptualised the idea, wrote the first draft. ABD, MK, JE, $\mathrm{RL}, \mathrm{SB}$, and WM, revised all the versions. All authors have read and approved the manuscript.

\section{Funding}

TB and RKK received funding from Life Support Foundation and The association of Anaesthetists.

\section{Availability of data and materials}

The data used in this manuscript is not readily available for public use. A request can be made to the department that own the data through the corresponding author on raphkazidule@gmail.com.

\section{Ethics approval and consent to participate}

Ethical Approval was granted from the College of Medicine Research and Ethics Committee number (P.07/18/2433).

\section{Consent for publication}

Individual consent was not possible as data was extracted retrospectively, but approval was included in the ethical approval (P.07/18/2433).

\section{Competing interests}

None Declared.

\section{Author details}

${ }^{1}$ Department of Anaesthesia and Intensive Care, Queen Elizabeth Central Hospital, Blantyre, Malawi. ${ }^{2}$ College of Medicine, University of Malawi, Blantyre, Malawi. ${ }^{3}$ Department of Emergencies and Critical Care, Oslo University Hospital, Oslo, Norway. ${ }^{4}$ Liverpool School of Tropical Medicine, Liverpool, UK. ${ }^{5}$ Malawi Liverpool Wellcome Trust Clinical Research Programme, Blantyre, Malawi. ${ }^{6}$ Department of Global Public Health, Karolinska Institutet, Stockholm, Sweden. ${ }^{7}$ Department of Infectious Diseases/ Venhälsan, Stockholm South Hospital, Stockholm, Sweden. ${ }^{8}$ Perioperative Medicine and Intensive Care, Karolinska University Hospital, Stockholm, Sweden.

Received: 22 April 2020 Accepted: 12 October 2020

Published online: 19 October 2020

\section{References}

1. O'NEILL J. Tackling drug-resistant infections globally: final report and recommendations; 2016

2. Deak D, Outterson $K$, Powers JH, Kesselheim AS. Progress in the fight against multidrug-resistant Bacteria? A review of U.S. Food and Drug Administration-approved antibiotics, 2010-2015. Ann Intern Med. 2016; 165(5):363-72.

3. WHO. Global Action Plan on Antimicobial resistance. 2015.

4. Huttner A, Harbarth S, Carlet J, Cosgrove S, Goossens H, Holmes A, et al. Antimicrobial resistance: a global view from the 2013 world healthcareassociated infections forum. Antimicrob Resist Infect Control. 2013;2(1):31.

5. Barbosa TM, Levy SB. The impact of antibiotic use on resistance development and persistence. Drug Resist Updat. 2000:3(5):303-11.

6. Wolff MJ. Use and Misuse of Antibiotics in Latin America. Clin Infect Dis. 1993;17(Supplement_2):S346-S51.

7. Levy SB. Antibiotic Resistance: Consequences of Inaction. Clin Infect Dis. 2001;33(Supplement_3):S124-S9.

8. Musicha P, Cornick JE, Bar-Zeev N, French N, Masesa C, Denis B, et al. Trends in antimicrobial resistance in bloodstream infection isolates at a large urban hospital in Malawi (1998-2016): a surveillance study. Lancet Infect Dis. 2017; 17(10):1042-52. 
9. de Kraker MEA, Davey PG, Grundmann H, group Bs. Mortality and hospital stay associated with resistant Staphylococcus aureus and Escherichia coli bacteremia: estimating the burden of antibiotic resistance in Europe. PLoS Med. 2011;8(10):e1001104 e.

10. Neidell MJ, Cohen B, Furuya Y, Hill J, Jeon CY, Glied S, et al. Costs of healthcare- and community-associated infections with antimicrobialresistant versus antimicrobial-susceptible organisms. Clin Infect Dis. 2012; 55(6):807-15.

11. Campion M, Scully G. Antibiotic use in the intensive care unit: optimization and De-escalation. J Intensive Care Med. 2018;33(12):647-55.

12. Kollef MH, Bassetti M, Francois B, Burnham J, Dimopoulos G, GarnachoMontero J, et al. The intensive care medicine research agenda on multidrug-resistant bacteria, antibiotics, and stewardship. Intensive Care Med. 2017:43(9):1187-97.

13. De Waele JJ, Akova M, Antonelli M, Canton R, Carlet J, De Backer D, et al. Antimicrobial resistance and antibiotic stewardship programs in the ICU: insistence and persistence in the fight against resistance. A position statement from ESICM/ESCMID/WAAAR round table on multi-drug resistance. Intensive Care Med. 2018;44(2):189-96.

14. Lucinda Manda-Taylor SM, Baker T. Critical care in Malawi: Ethics of beneficence and Justice. Malawi Med J. 2017;29(3):268.

15. BRB WTMG, Bultemeir K. Challenges Encountered by Intensive care nurses in Meeting Patients' Families Needs in Malawi. Afr J Nurs Midwife. 2011;13(2): 92-102.

16. Remschmidt C, Schneider S, Meyer E, Schroeren-Boersch B, Gastmeier P, Schwab F. Surveillance of antibiotic use and resistance in intensive care units (SARI). Dtsch Arztebl Int. 2017;114(50):858-65.

17. Johnston D, Khan R, Miot J, Moch S, Deventer Y, Richards G. Usage of antibiotics in the intensive care units of an academic tertiary-level hospital. South Afr J Infect Dis. 2018;33(4):1-8.

18. Labi A-K, Obeng-Nkrumah N, Sunkwa-Mills G, Bediako-Bowan A, Akufo C, Bjerrum S, et al. Antibiotic prescribing in paediatric inpatients in Ghana: a multi-centre point prevalence survey. BMC Pediatr. 2018;18(1):391.

19. MOH. The Malawi Standard Treatment Guidelines (MSTG) : Incorporating Malawi Essential Medicines List. 2015.

20. Mali NB, Deshpande SP, Tullu MS, Deshmukh CT, Gogtay NJ, Thatte UM. A prospective antibacterial utilization study in pediatric intensive care unit of a tertiary referral center. Ind J Crit Care Med. 2018;22(6):422-6.

21. Silva CD, Silva JM. Strategies for appropriate antibiotic use in intensive care unit. Einstein (Sao Paulo, Brazil). 2015;13(3):448-53.

22. Axente C, Licker M, Moldovan R, Hogea E, Muntean D, Horhat F, et al. Antimicrobial consumption, costs and resistance patterns: a two year prospective study in a Romanian intensive care unit. BMC Infect Dis. 2017; 17(1):358.

23. Zhang $Y Z$, Singh S. Antibiotic stewardship programmes in intensive care units: why, how, and where are they leading us. World J Crit Care Med. 2015;4(1):13-28.

24. Zhang ZG, Chen F, Ou Y. Impact of an antimicrobial stewardship programme on antibiotic usage and resistance in a tertiary hospital in China. J Clin Pharm Ther. 2017:42(5):579-84.

25. Mula CT, Human N, Middleton L. An exploration of workarounds and their perceived impact on antibiotic stewardship in the adult medical wards of a referral hospital in Malawi: a qualitative study. BMC Health Serv Res. 2019; 19(1):64.

26. Luyt CE, Brechot N, Trouillet JL, Chastre J. Antibiotic stewardship in the intensive care unit. Crit Care. 2014;18(5):480.

27. Allison MG, Heil EL, Hayes BD. Appropriate antibiotic therapy. Emerg Med Clin North Am. 2017;35(1):25-42.

28. Bassetti M, Poulakou G, Timsit J-F. Focus on antimicrobial use in the era of increasing antimicrobial resistance in ICU. Intensive Care Med. 2016;42(6): 955-8.

29. Sampane-Donkor E, Badoe EV, Annan JA, Nii-Trebi N. Colonisation of antibiotic resistant bacteria in a cohort of HIV infected children in Ghana. Pan Afr Med J. 2017;26:60.

\section{Publisher's Note}

Springer Nature remains neutral with regard to jurisdictional claims in published maps and institutional affiliations.

Ready to submit your research? Choose BMC and benefit from:

- fast, convenient online submission

- thorough peer review by experienced researchers in your field

- rapid publication on acceptance

- support for research data, including large and complex data types

- gold Open Access which fosters wider collaboration and increased citations

- maximum visibility for your research: over $100 \mathrm{M}$ website views per year

At BMC, research is always in progress.

Learn more biomedcentral.com/submissions 